\title{
Changement institutionnel, légitimation et politiques scolaires : le cas de la lutte contre le redoublement en Belgique francophone \\ Institutional Change, Legitimating and Education Policies : The Case of the Fight against Staying Down in French-Speaking Belgium
}

\author{
Hugues Draelants
}

Volume 40, numéro 1, printemps 2008

Les nouvelles politiques d'éducation et de formation

New Policies in Education and Training

URI : https://id.erudit.org/iderudit/019475ar

DOI : https://doi.org/10.7202/019475ar

Aller au sommaire du numéro

Éditeur(s)

Les Presses de l'Université de Montréal

ISSN

0038-030X (imprimé)

1492-1375 (numérique)

Découvrir la revue

Citer cet article

Draelants, H. (2008). Changement institutionnel, légitimation et politiques scolaires : le cas de la lutte contre le redoublement en Belgique francophone. Sociologie et sociétés, 40(1), 119-141. https://doi.org/10.7202/019475ar

\section{Résumé de l'article}

Cet article invite à lire les politiques scolaires comme des entreprises de changement institutionnel. L'approche cognitive adoptée conduit à placer la légitimation au coeur du processus de changement institutionnel. L'analyse est menée à partir de l'étude d'une politique de lutte contre le redoublement en Belgique francophone. Ce cas, qui s'est soldé par un échec relatif, illustre les limites et la complexité de toute entreprise politique de transformation institutionnelle de l'école. Alors que la réforme s'appuie sur l'expertise pédagogique et économique pour légitimer la suppression du redoublement, l'enquête montre que le changement institutionnel ne résulte pas mécaniquement de la perception de dysfonctionnements, mais nécessite le consentement des acteurs scolaires locaux, dans la mesure où le changement se joue de façon décisive dans les établissements scolaires et dans les classes. À cet égard, l'enquête souligne l'importance, tant théorique que pratique, de l'encastrement institutionnel du redoublement, encastrement qui mine la légitimité pragmatique de la réforme sur le terrain. 


\section{Changement institutionnel, légitimation et politiques scolaires : le cas de la lutte contre le redoublement en Belgique francophone}

\section{HUGUES DRAELANTS}

Observatoire sociologique du changement, Sciences Po / CNRS, FNRS / UCL / Girsef

1, Place Montesquieu

1348 Louvain-la-Neuve, Belgique

Courriel: hugues.draelants@uclouvain.be

$\mathrm{L}$ A LITTÉRATURE SUR LES POLITIQUES SCOLAIRES est une littérature abondante, aux démarches et méthodes variées. Elle puise ses sources du côté des historiens, des sociologues, des anthropologues ou encore des politistes. Si l'éclatement des perspectives d'analyse qui la caractérise peut être vu comme le gage d'une indéniable richesse, il est néanmoins regrettable de constater que peu de travaux se sont efforcés d'élaborer des modèles. Au terme d'un bilan des études consacrées aux politiques d'éducation, van Zanten souligne ce déficit théorique: ce qui manque cruellement pour se repérer dans cet univers, ce sont moins des analyses détaillées des réformes ou initiatives menées que des cadres et des outils pour penser les articulations entre les lois, les dispositifs, les intentions et les réalisations (van Zanten, 2004).

La discipline de l'analyse des politiques publiques (Muller et Surel, 1998) semble prédisposée à jouer un rôle heuristique à cet égard, puisque l'enseignement constitue par définition un domaine de l'action publique. En pratique, les spécialistes de l'analyse des politiques publiques ne témoignent guère d'intérêt pour le champ scolaire. Par ailleurs, les chercheurs en éducation qui s'interrogent sur les politiques éducatives ignorent, à quelques exceptions près, la discipline de l'analyse des politiques publiques ou du moins ses développements les plus récents. La méconnaissance réciproque prévaut donc largement. Toutefois, la situation évolue. Ces dernières années, plusieurs sociologues 
de l'éducation ont contribué à élaborer une analyse spécifique des politiques publiques d'éducation et ont mis sur pied des programmes de recherche en ce sens (Van Haecht, 1998; Derouet, 2000; Maroy et Dupriez, 2000; van Zanten, 2004; Buisson-Fenet, 2007). Notre travail s'inscrit dans cette tendance.

Afin de construire notre grille d'analyse, nous avons retenu certains des référents théoriques émergeant pour constituer une sociologie de l'action publique en éducation: ils relèvent d'une part de l'analyse des politiques publiques — en particulier dans sa version dite «cognitive» — et, d'autre part, de divers néo-institutionnalismes — singulièrement sous leur variante sociologique. Les «approches cognitives» des politiques publiques désignent les travaux qui insistent sur le poids des éléments de connaissance, des idées, des représentations ou des croyances sociales dans l'élaboration des politiques (Boussaguet et al., 2004). De manière générale, les différents courants institutionnels posent quant à eux «la nécessité de penser le rôle des médiations entre structures sociales et comportements individuels pour comprendre ces derniers et leurs formes collectives d'expression. Ces médiations sont précisément les institutions» (Théret, 2000). L'institutionnalisme sociologique (Powell et DiMaggio, 1991; Scott, 1995) se distingue par l'accent mis sur la dimension cognitive du processus d'institutionnalisation. Selon cette conception, les institutions influencent le comportement en fournissant des schémas, des catégories et des modèles cognitifs indispensables à l'action dans la mesure où ils permettent d'interpréter le monde et le comportement des autres acteurs.

Des liens solides sont déjà établis dans la littérature entre analyse des politiques publiques et néo-institutionnalismes (Hall et Taylor, 1997). Les rapprocher ne constitue pas en soi une innovation. L'apport envisagé consiste à proposer une articulation inédite en vue d'esquisser une théorie «à moyenne portée» du changement ou du maintien institutionnel en éducation. Le point de départ de notre raisonnement est le suivant: toute politique publique éclaire le contexte et l'objet de son intervention sous un jour particulier, portant donc en elle une disqualification implicite des autres politiques possibles et, généralement, une délégitimation explicite des interprétations concurrentes de la réalité, des alternatives proposées ou non et en définitive des institutions existantes, alors jugées inadaptées ${ }^{1}$. Il en découle que l'on peut, selon nous, appréhender les politiques publiques comme des entreprises de changement institutionnel, au sens cognitif du terme. L'opérationnalisation de cette proposition théorique se construit autour des notions de légitimation/délégitimation conçues comme des actions indispensables au cœur du processus de changement institutionnel ${ }^{2}$.

1. En éducation, c'est du moins le cas pour les réformes qui portent sur les contenus et les modalités d'enseignement ou d'évaluation, c'est-à-dire celles qui visent à transformer le travail des enseignants.

2. L'orientation théorique privilégiée nous conduit non seulement à centrer l'analyse sur l'étude des institutions immatérielles et des processus cognitifs de changement institutionnel, mais aussi à placer la question de la légitimité au cœur du processus de changement institutionnel. Cette orientation cognitive ne doit pas occulter le fait que l'institution n'existe pas uniquement sous une forme cognitive. Les schèmes cognitifs institués s'objectivent dans des structures spatiales et temporelles, des règlements, des dispositifs d'objets qui en constituent le pendant matériel. Ces dimensions ne sont pas ignorées. Cependant, notre modèle d'analyse s'avère moins opérationnel pour les saisir. 
L'objectif de cet article est donc de contribuer à la construction et à la diffusion de repères théoriques en proposant une réflexion organisée autour d'une problématique sociologique ancienne, celle du changement institutionnel. La présentation est structurée en trois parties. Chaque partie développe un aspect du processus de changement institutionnel: 1) sa source;2) son cœur; 3) ses effets. La réflexion théorique est systématiquement nourrie par des analyses empiriques tirées de l'étude d'une politique de lutte contre le redoublement initiée en 1994 et connue sous le nom de «réforme du premier cycle (ou degré) de l'enseignement secondaire $»^{3}$ (Draelants, 2006). Le cas de cette réforme s'avère au moins doublement pertinent pour illustrer notre problématique théorique: premièrement, cette tentative de changement institutionnel s'est soldée par ce qui peut apparaître comme un échec relatif, si l'on compare les résultats effectifs de la réforme à ses effets escomptés. Ce cas critique présente ainsi l'avantage de mettre en lumière toute une série d'obstacles au changement, obstacles de nature institutionnelle, insuffisamment pris en compte par l'action publique. Deuxièmement, cette réforme, loin d'être un cas unique, s'avère en fait emblématique du déficit de légitimité dont souffrent la plupart des réformes éducatives entreprises ces quinze dernières années en Belgique francophone, dès lors qu'elles touchent à la pédagogie (Mangez, 2002; Frenay et Maroy, 2004; Van Campenhoudt et al., 2004; Dupriez et Cornet, 2005).

Notre propos privilégie la dimension interprétative des résultats: il s'agit d'abord de montrer la pertinence heuristique et les implications théoriques qui découlent de l'étude d'une politique d'éducation comme processus de changement institutionnel. Les résultats empiriques sont donc ici minimalement présentés. Autre précision utile: cette réflexion, bien que menée à partir du cas belge, est susceptible d'aider à comprendre ce qui se passe dans d'autres contextes. D'autres systèmes éducatifs francophones font en effet l'objet de réformes similaires. Désormais, dans un contexte de massification scolaire étendue, les politiques éducatives menées en Europe et en Amérique du Nord durant les années 1990 et 2000, notamment dans les régions francophones (France, Québec, Canton de Genève, Communauté française de Belgique) cherchent à répondre aux problèmes qualitatifs que pose l'accès généralisé à l'enseignement: gestion des «nouveaux publics", modification du curriculum, des programmes de cours, des pratiques pédagogiques enseignantes et des structures organisationnelles des établissements.

\section{I. À la source du processus de CHANGEMENT institutionNel}

\section{Définition}

Un processus de changement institutionnel consiste en un double mouvement qui, théoriquement, suppose d'une part une désinstitutionnalisation et d'autre part une

3. À laquelle nous avons consacré notre thèse de doctorat, soutenue le 30 septembre 2006 à l'Université de Louvain. 
réinstitutionnalisation ${ }^{4}$. Le processus peut se faire de façon continue ou par étapes successives. Diverses conditions sociales (crise économique, sociale, technologique, etc.) peuvent constituer des «secousses précipitantes» de la désinstitutionnalisation, mais le processus nécessite simultanément l'intervention d'acteurs de changement institutionnel.

Nous suivons ici Greenwood, Suddaby et Hinings (2002) qui proposent un modèle non isomorphique du changement institutionnel. Une telle perspective a pour avantage de ne pas réduire la question de la légitimation à celle de l'imitation ${ }^{5}$, de mettre l'accent sur le fait que désinstitutionnalisation et réinstitutionnalisation sont le fruit d'actions et d'acteurs de changement, ne découlant pas seulement de causes dont l'apparition serait spontanée, comme des dysfonctionnements. Pour désigner les acteurs qui bénéficient de certains arrangements institutionnels et qui mobilisent des ressources en vue de transformer les institutions ou d'en créer de nouvelles, nous parlons d'entrepreneurs institutionnels (DiMaggio, 1988). Ces derniers contribuent à établir la légitimité de nouvelles institutions: il s'agit de fonder leur légitimité cognitive, morale et pragmatique, ce qui constitue une étape cruciale dans la réinstitutionnalisation.

La définition adoptée nous éloigne également d'une perspective dialectique du changement institutionnel (Seo et Creed, 2002), selon laquelle toute institution contiendrait en elle-même ses limites, ses contradictions internes, sous-produits du processus d'institutionnalisation. Celles-ci constituent dans ce cadre les forces fondamentales qui, à long terme et sous certaines conditions de mobilisation ou d'action collective, conduisent au changement institutionnel. Cependant, il existe également des sources intentionnelles de changement, des processus politiques volontaires de désinstitutionnalisation. L'État est en effet le premier des entrepreneurs de changement. Ces processus politiques supposent un travail de délégitimation et sont liés à la multiplicité des modèles institutionnels existants. Ce sont ceux dont nous traiterons ici, notre thèse étant qu'on peut lire les politiques d'éducation comme des entreprises de changement institutionnel.

\section{Le contexte pédagogique et économique qui va précipiter la réforme}

À l'origine de la réforme étudiée se trouvent plusieurs événements déclencheurs qui vont se combiner et s'articuler favorablement avec une intention politique de réforme du premier cycle de l'école secondaire. Un indicateur au moins va jouer ici un rôle crucial: il s'agit du taux de retard scolaire qui en regard des comparaisons internationales apparaît extrêmement élevé en Communauté française, comme le relèvent plu-

4. Selon cette perspective, il est impensable de parler de « déclin institutionnel » (Dubet, 2002) sans s'interroger sur la réinstitutionnalisation ou construction de nouvelles institutions. Les deux processus sont inséparables. Au déclin ne peut succéder le vide, l'institutionnalisation de nouvelles formes est inévitable.

5. L'explication sous-jacente à la thèse de l'imitation, que l'on trouve dans les modèles isomorphiques du changement institutionnel, est que les nouvelles règles et idées acquièrent de la légitimité si elles sont adoptées par des « autres exemplaires », dont elles conditionnent la réussite aux yeux de tous. Les organisations s'imitent parce qu'elles anticipent des bénéfices similaires. 
sieurs rapports consacrés à la question de l'échec scolaire, dont les résultats sont amplifiés par la presse de l'époque. Ainsi, les «données du problème», telles qu'exposées alors par le pédagogue Marcel Crahay, comparent les taux de retard scolaire enregistrés à l'école primaire dans treize pays européens. On constate à la lecture des chiffres que le retard scolaire est un phénomène très variable, dans certains cas inexistant (Danemark, Suède, Royaume-Uni), quasi inexistant (Irlande, Grèce, Italie) ou encore statistiquement significatif (Luxembourg, Espagne, France, Portugal, Belgique). Avec un taux de $16,9 \%$, la Belgique, commente Crahay, «a le triste privilège de présenter le taux de retard scolaire le plus élevé de l'Europe des Douze, mais aussi très probablement de l'Europe entière». Au niveau secondaire, ajoute-t-il, «la situation est tout aussi alarmante»: «En Communauté française de Belgique, $43,1 \%$ des étudiants de première année du secondaire ont un an de retard. En fin de sixième, ils sont 57,6\%.»C'est donc une minorité qui parcourt les douze années du primaire et du secondaire «à l'heure» (Crahay, 1992). L'école en Communauté française est alors considérée comme «gangrenée» par l'échec scolaire, problème jugé d'autant plus crucial qu'il est en lien avec l'efficacité (le niveau scolaire moyen), l'efficience (l'échec coûte énormément), l'égalité (tout le monde n'est pas touché de la même manière) et, in fine, l'équité (la justice) des systèmes scolaires.

Un autre élément contextuel, de nature conjoncturelle, va s'avérer décisif dans l'établissement de la réforme: il s'agit des difficultés financières de la Communauté française, confrontée au début des années 1990 à un déficit budgétaire de plus en plus intenable compte tenu de l'extrême rigidité du mode de financement des Communautés. Le secteur de l'enseignement qui absorbe à lui seul $78 \%$ du budget de la Communauté française est dès lors prioritairement concerné. Les économistes de l'Institut de recherches économiques et sociales (IRES) de l'Université de Louvain montrent, dans la Radioscopie de l'enseignement (1992), qu'il est possible de réaliser des économies significatives en supprimant l'échec. Selon l'IRES, le gonflement de la population scolaire imputable au redoublement s'élève au minimum à $8,17 \%$, soit environ 50000 élèves, ce qui équivaut à une classe d'âge complète ajoutée à l'effectif scolaire.

Les événements qui ont contribué à remettre en question l'organisation de la scolarité au début du secondaire s'avèrent en fait assez représentatifs des phénomènes habituellement observés dans la genèse des problèmes publics (Kingdon, 1984). Ces circonstances ne constituent que des prémices à la définition du problème public. Les éléments initiaux vont en effet être (re)définis par la mise en forme de récits et la production de discours éventuellement concurrents sur le même phénomène, compte tenu de la position sociale des différents acteurs concernés. Ces éléments ne déterminent rien par eux-mêmes, c'est davantage leur réception et leur usage par les acteurs concernés, publics ou privés, qui importe. On peut parler de déclencheurs ou de secousses précipitantes. Alors que les conclusions des rapports d'expertise relayées par les médias publicisent et dramatisent les taux élevés d'échec scolaire ou de redoublement, le contexte de crise économique va précipiter le mouvement de réforme au double sens du terme: il va le provoquer et le hâter. Pédagogues et économistes se rejoi- 
gnant dans la critique du redoublement, les responsables politiques, contraints par l'étroitesse des marges budgétaires de mettre en place toute mesure susceptible de rationaliser le fonctionnement du système, vont rapidement esquisser une politique de lutte contre le redoublement. Ainsi, dès octobre 1992, le nouveau ministre de l'Éducation Elio Di Rupo soumet à concertation un projet de lutte contre l'échec scolaire au premier cycle. La mise en œuvre de l'école en cycle avec interdiction de redoublement se fera finalement d'abord au premier cycle du secondaire plutôt qu'au primaire, comme cela avait été préconisé par les pédagogues afin de préparer les enseignants. Ce calendrier, peu cohérent d'un point de vue pédagogique, témoigne d'une grande hâte à réaliser le projet et montre que la réforme s'inscrit d'abord dans un plan d'austérité. Rarement mise en avant, cette rationalité économique n'est cependant pas niée par le ministre: "C'est au seuil du secondaire que l'échec sévit le plus; si l'on veut faire des économies, c'est là qu'il faut agir en priorité» (Le Soir, 21 juin 1994).

\section{Un processus conflictuel}

Le processus de changement institutionnel résulte de deux mouvements à la fois opposés et interreliés: d'une part, le travail de légitimation des «institutions» nouvelles (par exemple, nouvelles règles du jeu ou vision transformée des «problèmes» qui se posent), allant de pair avec la délégitimation des institutions existantes; d'autre part, la délégitimation des nouvelles «institutions» parallèlement à la légitimation des anciennes institutions. Des luttes pour promouvoir et imposer une certaine construction sociale de la réalité peuvent donc se produire entre acteurs favorables et acteurs opposés au changement institutionnel. Les deux constructions qui s'affrontent n'ont pas le même statut institutionnel: l'une a la force de l'évidence et n'est pas vue comme une construction, l'autre apparaît comme un produit humain, d'autant plus incongru que l'ancien est évident et ancré dans les routines. Les facteurs d'inertie inhérents à l'institution font en sorte que, même lorsque divers éléments du contrôle global ont évolué, le groupe reste soumis à des influences du passé auxquelles il demeure affectivement attaché, car c'est à partir d'elles qu'il s'identifie (Remy et al., 1978). Ainsi, la force des groupes de défense de l'ordre établi est de pouvoir s'appuyer sur des éléments concrets de nature à rapidement couper court à tout tâtonnement engendrant l'insécurité.

\section{Controverses et oppositions à la réforme}

En septembre 1994, toutes les écoles d'enseignement secondaire sont tenues d'organiser le premier «degré» en cycle et sans redoublement. La réforme est dans l'ensemble mal accueillie par le monde enseignant. Compte tenu de la crise budgétaire, l'aspect pédagogique de la réforme est volontiers perçu comme un vernis masquant la motivation économique. La réforme fait l'objet de deux grandes critiques: la première, portée par différents acteurs collectifs (opposition politique, syndicats enseignants, associations de parents), stigmatise la précipitation et le manque de moyens associés au projet gouvernemental, tout en s'accordant sur la condamnation pédagogique du 
redoublement. Ce «récit de politique publique» (Radaelli, 2000) discrédite donc les conditions de mise en œuvre de la réforme, en d'autres termes sa légitimité pragmatique. En ce sens, la controverse soulève d'emblée des enjeux importants et des questions délaissées par les responsables politiques. L'autre critique discernable à l'époque est celle de la crainte du nivellement par le bas. Le désaccord est ici idéologique. Il se traduit par un rejet de l'«école de la réussite», objectif gouvernemental invoqué. Les tenants de ce discours élitiste refusent le postulat d'éducabilité généralisée qui sous-tend la réforme, les propos sont imprégnés d'une conception innéiste de l'intelligence. Cette critique radicale est le fait d'individus - enseignants, parents d'élèves ou citoyens s'exprimant non pas collectivement mais en leur nom propre. Seule exception, le député libéral Pierre Hazette, qui va rapidement s'imposer comme le responsable politique le plus farouchement opposé à la réforme du premier degré, initiée par les socialistes. Devenu ministre de l'Éducation à l'occasion d'un changement de majorité consécutif aux élections de 1999, il formule dès son arrivée au pouvoir le souhait de modifier ladite réforme. Sa priorité, dit-il, est de réhabiliter la «loi de l'effort». Sa contre-réforme sera mise en place en 2001.

Nous allons revenir sur le processus de légitimation de cette révision. En ce qui concerne son contenu, on peut dire que, sans réhabiliter stricto sensu le recours au redoublement, la contre-réforme de 2001 signe l'abandon du principe de passage automatique de classe pour une mesure moins ambitieuse, mais jugée plus praticable, qui consiste en la possibilité laissée aux établissements d'aménager au sein même du cycle un curriculum spécial pour les élèves en échec (une «première année complémentaire»).

Certains acteurs, soutenant la réforme du premier degré depuis 1994, dénoncent ce projet, l'estimant en porte-à-faux avec l'esprit initial de la réforme et y voyant un retour à une forme déguisée de redoublement. Les statistiques disponibles aujourd'hui (tableau 1) tendent à confirmer le bien-fondé de cette vision. Les enseignants semblent avoir interprété le message du ministre comme un retour en arrière, et dès 2002 on constate que le taux de redoublement redevient équivalent à celui de la période antérieure à la réforme du premier degré. Le taux de redoublement en fin de deuxième, qui avait fortement progressé par suite de l'interdiction de redoublement au sein du cycle, retrouve quant à lui un niveau proche de celui de 1995. Manifestement, la "première année complémentaire» ne remplit pas ou seulement imparfaitement sa fonction de remédiation.

Tableau 1 Évolution des taux de redoublement (\%) en première et en deuxième année d'enseignement secondaire, entre 1992-1993 et 2006-2007

\begin{tabular}{|c|c|c|c|c|c|c|c|c|c|c|c|c|c|c|c|}
\hline & $92-93$ & $93-94$ & $94-95$ & $95-96$ & $96-97$ & $97-98$ & $98-99$ & 99-०0 & O০-01 & O1-02 & $\mathrm{O} 2-03$ & $03-04$ & 04-05 & $05-06$ & $06-07$ \\
\hline $\begin{array}{l}\text { 1 A + } \\
\text { compl. }\end{array}$ & 10,6 & 10,2 & 9,0 & 1,9 & 1,8 & 1,4 & 1,4 & 1,6 & 1,4 & 2,2 & 9,3 & 10,4 & 11,6 & 11,9 & 12,1 \\
\hline $\begin{array}{l}2 \mathrm{C}+ \\
\text { compl. }\end{array}$ & 11,5 & 10,5 & 8,9 & 7,4 & 6,2 & 7,1 & 7,5 & 8,5 & 9,2 & 10,1 & 11,9 & 8,5 & 8,8 & 8,9 & 9,7 \\
\hline
\end{tabular}

Source: Ministère de la Communauté française de Belgique/Etnic, 2007. 
Ceci laisse entrevoir que le processus de changement institutionnel est ouvert et incertain. Sa réussite n'est nullement assurée, et cela pour deux raisons. D’une part, on l'a vu, l'action publique est susceptible de se heurter à des stratégies de "nonchangement» institutionnel ou de réinstitutionnalisation. D'autre part, l'institutionnalisation de nouvelles règles suppose un difficile travail de légitimation. À cet égard, l'analyse suggère, nous allons le voir, que les responsables politiques n'ont guère soigné les conditions de recevabilité de la réforme.

\section{AU CEUR DU PROCESSUS DE CHANGEMENT INSTITUTIONNEL}

\section{Légalité et légitimité}

Comme l'a montré Max Weber, la croyance en la légalité représente la plus courante des formes de légitimité dans la société moderne. D’ailleurs, certaines définitions tendent à assimiler la légitimité à la légalité, c'est-à-dire la conformité à la loi (Ferréol, 1995). Ce n'est pas la voie suivie ici. Au contraire, conformément à une perspective sociologique et non juridique, nous considérons que la légitimité de l'action publique n'est pas réductible à la question de la légalité et du respect des procédures démocratiques de gouvernement. La légitimation d'un pouvoir est de fait, non de droit (Boulad-Ayoub, 2003). Si la légitimité des politiques d'éducation au sens de leur légalité ne pose guère problème, en revanche, le fait que la réforme ait force de loi ne suffit pas pour convaincre les enseignants de son bien-fondé, un processus de conversion et d'adhésion est nécessaire. Dans l'optique du néo-institutionnalisme sociologique, la question fondamentale est précisément de savoir ce qui confere de la légitimité à certains arrangements institutionnels plutôt qu'à d'autres (Hall et Taylor, 1997). Pour leur part, les spécialistes de l'analyse des politiques publiques soulignent que ces dernières n'existent pas sans toute une série de dispositifs de légitimation qui permettent leur acceptation (Hassenteufel et Smith, 2002).

La légitimation n'est pas un acte momentané, mais un processus qui s'inscrit dans la durée et qui nécessite une action répétée (Greenwood, Suddaby et Hinings, 2002). Dans le cadre de la recherche menée, nous avons analysé le travail de légitimation/délégitimation de la réforme du premier degré à différents moments et étapes du cycle politique, sur diverses scènes sociales et avec différents acteurs, entrepreneurs de changement. L'ambition est moins de proposer un examen exhaustif des opérations de construction de la légitimité que d'illustrer leur diversité à partir d'un découpage significatif. Nous envisagerons donc celles-ci d'une part à l'étape de la conception de la réforme sur la scène du parlement et d'autre part à l'étape de la mise en œuvre sur les scènes des établissements scolaires et des classes ${ }^{6}$.

6. Pour ce faire, nous mobiliserons des résultats issus de deux enquêtes empiriques : l'une porte sur les débats et les controverses relatifs aux politiques scolaires concernant le premier degré, ainsi que sur les usages politiques et stratégiques des arguments pédagogiques dans ces discussions. Cette étude repose sur l'analyse d'un vaste corpus de textes issus de deux types d'archives. Une première source documentaire est constituée par l'ensemble des articles parus entre 1993 et 2003 dans le journal Le Soir — premier quotidien 


\section{Conception et légitimation}

Début 2000, le ministre Hazette annonce un projet de décret corrigeant la réforme de 1994, dans l'intention d'assouplir le non-redoublement au début du secondaire. Ces intentions de «réformer la réforme» du premier degré affichées, s'ensuit une vive controverse avec le parti Écolo, un des partenaires de la majorité et en particulier du ministre Jean-Marc Nollet (responsable de l'enseignement fondamental). Deux récits de politique publique s'affrontent. Dans le récit de Pierre Hazette, tous les problèmes que rencontrent les enseignants pour appliquer la réforme de 1994 sur le terrain ont pour seule cause le passage automatique de première en deuxième année. Les écologistes considèrent le projet du ministre comme un retour en arrière, ce qui constitue l'argument central du second récit. Selon eux, si, sur papier, l'école en cycle reste la ligne de conduite, dans les faits, ce projet permet aux écoles de la contourner. Pour Écolo, c'est donc la "philosophie de l'école en cycle» qui est en jeu.

Dans un premier temps, le conflit se cristallise, le ministre Hazette refuse de reculer. Toutefois, il se retrouve rapidement isolé; le rapport de force au sein du gouvernement ne lui est pas favorable car le ministre Nollet a le soutien des socialistes, également membres de la coalition. C'est finalement le recours à l'expertise qui permet de sortir de la controverse. Un ministre socialiste propose en effet une médiane: évaluer le nonredoublement avant de l'assouplir.

Le ministre procède alors entre autres à une «consultation» des enseignants du premier degré par voie d'un questionnaire adressé aux directions? ${ }^{7}$. Quelques mois plus tard, le ministre Hazette remet à l'agenda son idée d'année complémentaire. Il affirme cette fois clairement et dans des termes fédérateurs que celle-ci ne sera pas « un duplicata de la première année mais bien un modèle de pédagogie différenciée» (Le Soir, 7 mars 2001). L'article premier de l'avant-projet de décret stipule que «l'année complémentaire ne peut en aucun cas constituer un redoublement de l'année antérieure». Il apaise ainsi les craintes des écologistes. La seconde mouture du projet Hazette permet donc de sortir de la controverse opposant les deux récits évoqués et constitue en ce sens un méta-récit qui aboutira au décret du 19 juillet 2001 (la réforme de la réforme).

de la presse belge francophone - à propos ou autour de la réforme. Une seconde source documentaire est formée de l'ensemble des comptes rendus intégraux des débats tenus entre les parlementaires à propos de la réforme au sein du Conseil de la Communauté française. La période considérée ici va de 1992 à 2001 . L'autre étude empirique dont nous rapporterons quelques résultats porte sur la réception et l'appropriation de la réforme au sein de trois établissements scolaires contrastés, et sur sa mise en œuvre par leurs enseignants dans les classes. Les trois établissements ont chacun fait l'objet d'une monographie dans le cadre de la recherche Reguleduc (pour une synthèse, voir Maroy, 2006) à laquelle nous avons participé en tant qu'enquêteur. Ce terrain a été réalisé durant l'année scolaire 2002-2003. Les données récoltées comprennent une cinquantaine d'entretiens, ainsi qu'une série d'observations ponctuelles. Nous avons également recueilli et exploité des documents internes aux établissements. Enfin, les données qualitatives ont été complétées et confrontées à un ensemble de statistiques sur ces écoles.

7. Cette démarche, vivement critiquée par un collectif de chercheurs belges en éducation, fut perçue comme s'apparentant en fait à un plébiscite sous couvert d'une évaluation scientifique. 


\section{La légitimation par les savoirs et les expertises pédagogiques... et ses limites}

Cet épisode illustre un résultat important de notre analyse, à savoir le recours politique aux connaissances et recherches en éducation à des fins de légitimation de la réforme du premier degré (Draelants, 2007). Le recours à l'expertise des pédagogues a été utilisé en 2001 pour dépasser les antagonismes au sein de la majorité, mais, nous l'avons évoqué, il a aussi servi en 1994 de stratégie politique pour donner sens à des rationalisations, pour légitimer des mesures difficiles et, in fine, pour maintenir la paix sociale à une époque de coupes sombres dans le budget de l'enseignement (Van Haecht, 2004).

Ce faisant, les responsables politiques ont converti les experts, de gré ou de force', en entrepreneurs de changement institutionnel. Néanmoins, ce n'est pas parce que le recours à l'expertise se généralise que l'action publique se fonde uniquement sur des connaissances «scientifiques». Le politique mobilise une pluralité de registres pour construire la légitimité de son action (les savoirs de terrain; le sens commun ou la «pédagogie du bon sens»; la profession de foi et l'affirmation de ses convictions politiques et personnelles; l'invocation d'« urgence» ou au contraire du droit à la lenteur; etc.). Par ailleurs, si le recours à la connaissance pédagogique présentée comme scientifique, par une sorte d'appel à la raison universelle, vise à fonder l'objectivité des débats et à dépasser le particularisme du discours idéologique, cela n'empêche toutefois pas la politisation. Le recours au pédagogique ne permet qu’une pseudo-dépolitisation des débats. Au-delà même des querelles quant à la manière d'interpréter le contenu d'un rapport scientifique ou quant au statut à lui accorder, la re-politisation systématique des échanges constatée montre que le discours politique, dans sa nature même, ne peut demeurer dans un registre expert (Le Bart, 1998).

L'analyse de l'évolution des débats parlementaires autour de la réforme du premier degré a par ailleurs fait apparaître un changement dans la façon de conduire et de légitimer l'action publique de réforme de l'enseignement. Alors qu'en 1994 le pouvoir politique privilégie le recours aux savoirs experts des pédagogues pour légitimer la réforme, entre 1997 et 2001 on assiste progressivement, sous la pression des enseignants, à la valorisation des savoirs d'expérience ou de terrain et à leur constitution en une forme d'expertise, ancrée dans la pratique professionnelle.

La pédagogie a en fait assez vite montré ses limites comme source de légitimation de la réforme. D’une manière générale, la scientificité des savoirs pédagogiques pâtit d'un statut épistémologique hybride. En effet, la pédagogie est à la fois science, art et philosophie de l'éducation. En conséquence, beaucoup de personnes se sentent autorisées à tenir un discours sur l'éducation: pour avoir été enfant ou en tant que parent, on «sait» ce qu'est l'éducation et comment il faut éduquer. Une catégorie sociale qui s'estime particulièrement en droit de revendiquer une compétence pédagogique est bien entendu celle des enseignants. On touche ici à la seconde limite des savoirs péda-

8. Certains chercheurs peuvent trouver un intérêt à jouer le jeu de l'expertise. Qui dit utilisation politique de la science dit, au moins potentiellement, stratégie politique de scientifiques (Javeau, 2001). 
gogiques comme source de légitimation de l'action politique: le recours politique à ces savoirs est volontiers perçu par les enseignants comme une atteinte à leur professionnalité. Ces dernières années, chez les enseignants de Belgique francophone, le sentiment d'être dépossédés de leur métier s'accompagne d'un dénigrement des experts jugés «déconnectés des réalités de terrain» et d'un rejet de leur «jargon pédagogique». Les syndicats vont d'ailleurs progressivement politiser la question pédagogique ${ }^{9}$ à travers la «dé-technicisation» des débats, ils revendiquent l'autonomie professionnelle des enseignants et leur intention de se réapproprier leur métier. Parallèlement au rééquilibrage entre savoirs experts et savoirs de terrain, on assiste aussi au renouvellement des rapports de pouvoir entre pédagogues universitaires et enseignants vis-à-vis du politique. Alors que l'expérience enseignante se mue en expertise de terrain, le rôle des chercheurs consiste de plus en plus, sans s'y réduire, à être à l'écoute du terrain et de sa «vérité».

\section{Légitimité morale, cognitive et pragmatique}

Il est heuristique d'opérer une distinction entre les trois types de légitimité nécessaires pour parvenir à une institutionnalisation complète: la légitimité morale, la légitimité cognitive et la légitimité pragmatique (Suchman, 1995) ${ }^{10}$. L'institutionnalisation de nouvelles idées suppose en théorie qu'elles acquièrent une légitimité morale ou en d'autres termes qu'elles correspondent aux prescriptions normatives dominantes. Ainsi, dans le cas de la réforme du premier degré, pour plaire aux partenaires de la majorité et parvenir à agréger des intérêts différents, le ministre Hazette a pendant un temps ${ }^{11}$ évité tout discours moralisateur, jugé peu recevable dans le contexte de l'époque. L'entreprise de changement institutionnel peut également s'appuyer sur des savoirs et des acteurs scientifiques, pourvoyeurs de légitimité cognitive. Pour autant, la légitimation de la réforme ne peut se résumer, selon nous, à avancer des idées justes moralement et scientifiquement. Les arrangements institutionnels promus doivent encore, en principe, démontrer leur supériorité fonctionnelle, ou légitimité pragmatique. Le processus de changement institutionnel implique donc une série de conditions de légi-

9. Cette évolution devient très nette à partir de 2003. En début d'année, dans un contexte d'élection législative imminente, les syndicats passent à l'attaque : l'imposition de méthodes pédagogiques conduit, estiment-ils, à désapproprier les enseignants de leur métier au profit des « gourous » de la pédagogie. En front commun, ils réclament donc un moratoire pédagogique et exigent que les réformes pédagogiques soient désormais soumises à l'aval des enseignants. Le lien avec la politique des cycles et de lutte contre le redoublement est explicite : les syndicats focalisent leurs critiques sur ces réformes.

10. Similairement, la mise en cause de la légitimité, la délégitimation, peut porter sur ces divers aspects.

11. Cependant, dans ses entrevues de l'époque à la presse, le message qu'il déclare adresser aux élèves, aux parents et aux enseignants à travers sa contre-réforme montre que ses convictions n'ont pas fondamentalement évolué depuis 1994 : «L'enseignement secondaire doit initier — progressivement mais de façon constante et déterminée - les adolescents au travail, à l'acceptation de l'effort et à l'accomplissement de leur potentiel. Il faut retrouver la loi du travail. On ne réussit pas sans effort. Pour avoir négligé ce message, le secondaire n'est plus au niveau de certaines nations qui nous sont proches » (Le Soir, 7 septembre 2001). 
timité à remplir, ce à quoi renvoie la distinction entre légitimité morale, cognitive et pragmatique. L'institutionnalisation est accomplie lorsque le nouveau mode de fonctionnement et les idées qui le sous-tendent deviennent des évidences, constituant alors un arrangement vu comme naturel et approprié.

L'analyse de l'entreprise politique de réforme du premier degré nous incite à penser que si la légitimité morale et la légitimité cognitive se construisent prioritairement en amont sur la scène publique, lors de la conception de la politique, en revanche, la légitimité pragmatique se construit largement en aval, à l'occasion de la mise en ouvre de la réforme sur toutes les scènes locales, dans les établissements scolaires. Notre enquête dans les établissements nous a par ailleurs permis de mettre en évidence l'importance primordiale de la légitimité pragmatique pour le devenir et la réussite de la réforme.

\section{Mise en œuvre et légitimation}

Le constat est banal mais il mérite d'être rappelé: les chefs d'établissement et les enseignants, contrairement aux analystes des politiques publiques, ne sont pas concernés en premier lieu par le système politique et les politiques éducatives. Les acteurs scolaires locaux ont quantité d'autres préoccupations journalières généralement plus significatives à leurs yeux. Dès lors, afin de comprendre tant les logiques d'action des chefs d'établissement et des enseignants que la manière dont ils perçoivent et mettent (ou non) en œuvre les réformes pédagogiques, il importe de référer leur comportement aux contextes d'action pertinents pour chacun de ces acteurs: l'établissement ou le niveau de l'organisation scolaire pour le chef d'établissement; la classe, espace intraorganisationnel partiellement autonome, pour l'enseignant. Chacune de ces scènes se caractérise par une configuration d'acteurs qui lui est propre et par des contraintes différentes qu'il s'agit de bien cerner afin de comprendre le sens local de la réforme et les tests de légitimité auxquels se heurte l'action publique.

\section{Acteurs pertinents et contraintes pour l'action au niveau de l'établissement}

La régulation politique (par exemple, les règles sur les modes de financement, la structuration et le mode d'organisation du système scolaire, les politiques publiques mises en œuvre) cadre et influence l'action des entités locales du système scolaire. Au-delà de la régulation politique, les actions des écoles sont partiellement conditionnées par les positions qu'elles occupent par rapport aux écoles environnantes. Cela est d'autant plus vrai en Belgique francophone où, conformément à la liberté permise en matière d'offre d'enseignement et de choix d'école, les établissements se font concurrence sur un "quasi-marché» scolaire, afin d'attirer un public suffisant, puisque du nombre d'élèves inscrits dépend le financement de l'école. Les logiques d'action sont également tributaires des réalités culturelles et (micro)politiques internes à l'établissement. Chaque école peut être analysée comme une organisation scolaire semblable à n’importe quelle autre par sa structure, mais unique par son histoire, par sa dynamique de fonction- 
nement, par les acteurs qui la composent. On peut considérer que ces réalités culturelles (valeurs, normes informelles, représentations partagées) et politiques (rapports de pouvoir à l'interne et modes de coordination locale entre acteurs) dotent l'établissement d'une identité organisationnelle spécifique. Les logiques d'action de l'établissement peuvent avoir pour visée de maintenir cette identité. Des tensions sont susceptibles d'apparaître entre ce qui est décrété au niveau législatif par la politique d'éducation et ce qui est inscrit, parfois de longue date, dans une identité d'établissement et qui définit un ordre local.

\section{Acteurs pertinents et contraintes pour l'action au niveau de la classe}

La mise en œuvre se joue sur la scène organisationnelle de l'établissement, mais elle se joue aussi sur la scène de la classe, où l'enseignant est prioritairement actif. Pour lui, la réalité des établissements, c'est d'abord la classe dans laquelle il passe l'essentiel de son temps à l'école. Bien qu'il existe une pluralité d'expériences du métier enseignant en fonction des conditions de travail assez inégales d'un établissement à l'autre, la «forme classe» que l'on retrouve dans tout établissement scolaire définit au moins un invariant du métier: la gestion de la classe. Le maintien de l'ordre dans la classe est nécessaire pour «faire cours». Le rapport aux élèves structure le travail enseignant et constitue le cœur de l'activité d'enseignement, bien que d'autres composantes de cette activité gagnent actuellement en importance (le rapport aux collègues, la formation et le développement professionnel, la participation à des tâches organisationnelles) (Cattonar, 2005). Plus que la classe, ce sont donc les élèves (présents dans la classe) qui représentent aux yeux des enseignants le «contexte» de travail le plus important.

\section{Test de légitimité de la réforme sur la scène de l'établissement}

L'analyse comparée de la réception et de la mise en œuvre de la réforme du premier degré dans trois établissements scolaires belges, contrastés en termes de position hiérarchique sur le quasi-marché et de projets pédagogiques, a révélé deux faits: d'une part, la lecture que font les établissements de la politique d'éducation varie considérablement d'une école à l'autre; d'autre part, cette lecture dépend des priorités et des projets locaux (logiques d'action de l'établissement), eux-mêmes liés à la position de l'établissement dans la hiérarchie scolaire locale, ainsi qu'à son identité.

Au niveau de l'établissement, la légitimité de la réforme dépend de la perception éventuelle de tensions entre la réforme et la ou les logiques dominantes de l'action locale. Lorsque la réforme est perçue comme une opportunité, au sens où elle vient renforcer ou légitimer les identités et les logiques d'action locales, les établissements ont tendance à s'engager dans la réforme, voire à la récupérer. À l'inverse, lorsqu'une réforme est perçue comme menaçante pour la position ou l'identité de l'établissement, celui-ci a tendance à l'ignorer, de manière à éviter de perturber son fonctionnement. Entre ces deux stratégies, idéales-typiques, faut-il le préciser, des stratégies intermédiaires sont possibles. Par exemple, les établissements peuvent adopter symbolique- 
ment la réforme sans que celle-ci n'influence en profondeur le travail de l'équipe éducative, en reprenant le langage officiel (comme les concepts centraux de la réforme) ou en rendant visibles les changements opérés dans le sens de la pression externe sans rendre compte des pratiques allant à son encontre (par exemple, en instaurant un programme de remédiation spécifique pour élèves en échec parallèlement à l'organisation implicite de classes de niveau).

Illustrons ces résultats par une description succincte des situations rencontrées dans les trois établissements étudiés ${ }^{12}$.

Au sein de l'Institut Sainte Madeleine, la représentation collective du public et de l'action éducative est traditionnellement marquée par une centration sur l'élève appréhendé dans sa globalité, et par une action éducative principalement expressive. L'établissement vise explicitement la réussite scolaire du plus grand nombre, veillant pour ce faire à s'adapter aux besoins du public et à s'investir dans le soutien des élèves en difficulté sur le plan scolaire ou comportemental. Sainte Madeleine s'apparente ainsi à une école spécialisée dans le soutien des élèves en difficulté, par opposition aux écoles plus élitistes peu sensibles à la remédiation scolaire. Cette logique d'action permet à l'école d'occuper une "niche» (Dupriez et Cornet, 2005) au sein du marché scolaire local. Dès lors, la logique d'action de Sainte Madeleine s'est vue légitimée et renforcée par la philosophie égalitariste qui sous-tend la réforme du premier degré d'une double manière: d'une part, en offrant à l'établissement scolaire un critère de légitimité externe et central qui assure la reconnaissance d'une culture locale à la marge du système et, d'autre part, en fournissant à l'équipe éducative des outils et des concepts pédagogiques (organisation en cycle, pédagogie de la réussite, remédiation) qui traduisent les intentions égalitaires locales sous la forme d'un langage reconnu par le législateur.

Dans l'Athénée Meunier, de l'avis des acteurs, le type d'élèves recrutés s'est un peu «dégradé» ces dernières années. En raison de fermetures d'écoles dans la commune voisine - l'une des plus pauvres de la région — et par le simple jeu de l'organisation des transports en commun, l'établissement a recueilli nombre d'élèves d'origine turque ou maghrébine habitant ces quartiers environnants. Le classement récent de l'établissement en discrimination positive reflète cette situation. Le préfet n'a pas l'impression de pouvoir faire grand-chose pour contrôler le type de public qui fréquente son établissement. Dès lors, face à cette évolution, la direction tente de préserver la réputation de son établissement — que l'on peut décrire comme une bonne école populaire — en mettant tout en œuvre pour éviter l'étiquette d'école «difficile» ou d'école «à problèmes ». De fait, l'école a connu quelques problèmes de discipline mais a rapidement réagi afin de préserver son équilibre organisationnel interne et sa réputation. Cette logique de maintien de l'ordre s'observe par le renvoi d'un nombre conséquent d'élèves, par l'installation de barrières sur tout le périmètre de l'école, par l'engagement d'un proviseur chargé de la discipline... La logique locale centrée sur la discipline et sur l'ordre éducatif rentre en tension avec la logique égalitariste de la réforme. La réaction de

12. Les noms des établissements sont modifiés afin de respecter l'anonymat. 
l'école consiste dès lors à contourner la réforme ou à pervertir son esprit initial dans la manière de l'appliquer, pour la mettre au service de la logique d'action de l'établissement. Cela se donne notamment à voir dans la mise en œuvre de l'«année complémentaire»: l'établissement a spécialement mis en place un cours d' "éducation à la citoyenneté» qui a pour objectif de rappeler à ces élèves les règles de base du «vivre ensemble» et de leur inculquer certaines normes disciplinaires.

Enfin, à l'Athénée Boileau, un établissement en position dominante et bénéficiant d'une grande réputation dans la région, la culture de l'établissement, qui valorise fortement l'élitisme et l'excellence académique, est également menacée par les buts des politiques éducatives récentes. En outre, ces dernières années, compte tenu de la baisse démographique, Boileau est confronté à un problème de recrutement, particulièrement au premier degré où une perte considérable d'élèves y met en péril les emplois enseignants. Dans ce contexte, la logique d'action de la direction consiste en une politique d'ouverture au premier degré. L'ouverture relative à ce niveau ne signe toutefois pas la disparition d'une logique élitiste qui reste très vivace dans les degrés supérieurs. De la sorte, le fonctionnement des deux premières années d'enseignement secondaire apparaît dissocié du fonctionnement général de l'école. La mise entre parenthèses de la logique élitiste au cours des deux premières années permet aussi à Boileau d'afficher une image plus légitime dans le contexte politique et pédagogique de l'«école de la réussite» tout en sauvegardant le fonctionnement sélectif dans les cycles supérieurs.

\section{Test de légitimité de la réforme sur la scène de la classe}

Les enseignants rencontrés n'apparaissent pas fondamentalement «résistants au changement». La grande majorité accepte sans rechigner de se remettre en question et de procéder à la mise en ouvre des réformes. Les nouvelles prescriptions pédagogiques sont alors testées à travers un processus d'essais et d'erreurs. Pour être acceptées, elles doivent vraiment montrer leur pertinence au quotidien (c'est-à-dire dans la pratique du métier en classe avec les élèves), prouvant alors qu'elles ne sont pas simple verbiage abstrait d'intellectuels. C'est le test que doit subir la réforme pour être reconnue comme légitime par une majorité d'enseignants. En l'absence d'une telle concrétisation, la neutralisation se renforce et l'effet de bon sens joue en faveur des formes établies, les modèles nouveaux semblent relever de bonnes intentions, mais quelque peu irréalistes. L'analyse montre que cela se produit dans de nombreux cas (dans la plupart des cas observés). Les enseignants se sentent alors libres d'adapter les réformes. Soulignons que la conservation par les enseignants d'un quant-à-soi pédagogique n'est pas uniquement une question pratique de mise en œuvre, c'est aussi plus largement un enjeu professionnel. Il s'agit pour eux d'affirmer leur autonomie vis-à-vis de l'État et de divers experts en pédagogie. Dans la mesure où ils sont sur le terrain, les enseignants ont le sentiment d'être les mieux placés, donc les plus compétents, afin d'estimer la faisabilité des réformes pédagogiques.

Les élèves ont ainsi un effet indirect important sur la mise en œuvre de la réforme. En l'occurrence, dans le cas de la réforme du premier degré, les élèves font plutôt office 
d'entrepreneurs de non-changement institutionnel. En 1994, à la suite de l'interdiction de redoublement au premier degré et de l'instauration d'un passage automatique de classe, les enseignants constatent que les élèves ne travaillent plus. La réforme est accusée d'engendrer un manque de sérieux de la part des élèves, la suppression du redoublement tuerait le sens de l'effort. Ceci entraîne des conséquences négatives pour les élèves: ceux qui n'ont pas travaillé, n’ayant pas saisi à temps l'importance de la première orientation au terme du premier degré, se retrouvent au pied du mur lorsqu'ils sont en échec, renvoyés vers les filières techniques et professionnelles. Les enseignants invoquent également des conséquences négatives sur leur propre travail: en l'absence d'une menace de redoublement, ils ont beaucoup de difficultés à motiver les élèves, mais également à tenir la classe et à maintenir leur autorité. D'où une évaluation très négative par les enseignants de la mesure de suppression du redoublement prise à l'occasion de la réforme de 1994, que nombre d'entre eux considèrent comme une «ineptie». Au fond, ce qui semble poser problème, ce que les enseignants déplorent, c'est le rapport instrumental au savoir et à la scolarité dont font preuve les élèves. Beaucoup travaillent d'abord pour la note plutôt que par intérêt intellectuel. La réforme se heurte à ce minimalisme.

\section{LES EFFETS DU PROCESSUS DE CHANGEMENT INSTITUTION NEL}

\section{Un processus ouvert et incertain}

On le voit, seule une analyse des processus locaux de mise en œuvre et de construction de la légitimité de l'action publique permet d'apprécier ce que produisent effectivement les politiques publiques. Rien ne garantit que la politique de changement institutionnel poursuivie par les autorités politiques va s'imposer, être acceptée et reconnue comme «vraie» par la majorité des acteurs du secteur. Il s'agit d'une question empirique.

La réforme a d'ailleurs connu un destin controversé et mouvementé: initiée en 1994, elle est, on l'a dit, révisée sept ans plus tard à l'initiative du ministre Hazette ( «réforme de la réforme», décret du 19 juillet 2001). En cause, la pertinence de sa mesure phare, à savoir la décision prise initialement de supprimer la possibilité de redoublement au sein du premier cycle et d'instaurer en lieu et place un dispositif de promotion automatique.

\section{L'encastrement institutionnel du redoublement}

L'attachement des enseignants au redoublement témoigne du caractère institutionnalisé de cette pratique. Comprendre les débats suscités par cette réforme suppose alors d'envisager le redoublement sous l'angle d'un dispositif organisationnel et pédagogique institutionnellement encastré, plutôt que sous celui d'une simple «décision administrative». L'influence des dispositifs organisationnels et pédagogiques est en effet médiatisée par les représentations socialement partagées qui attribuent un certain sens, 
voire une fonction particulière, aux dispositifs du système. Avec le temps, ces constructions sociales s'institutionnalisent, ce qui rend leur transformation difficile même lorsqu'il y a une détermination collective à les modifier. Rappelons à cet égard qu'une caractéristique des faits institutionnalisés est de constituer en eux-mêmes des barrières à leur propre réforme (Powell et DiMaggio, 1991). Même modifiés ou supprimés, les faits institutionnalisés ne perdent pas immédiatement de leur influence (Archer, 2004).

Les réactions négatives recueillies dans les établissements par rapport à l'interdiction du redoublement montrent que celui-ci servait et sert toujours en Communauté française (là où il n'est pas interdit) à assumer une série de fonctions latentes qui lui confèrent une légitimité fonctionnelle ou pragmatique auprès des enseignants (Draelants, 2008). Ces fonctions latentes sont liées à des règles de fonctionnement institutionnalisées au sein des classes, des établissements, des espaces scolaires locaux et du système scolaire en Communauté française. Règles de fonctionnement et modes de régulation institutionnalisés dont les acteurs du système se font les vecteurs. Le sens du redoublement n'est donc pas indépendant des autres institutions du système scolaire belge francophone qui «réagissent» à l'entreprise politique de réforme et ont tendance à fonctionner comme des contraintes fortes, voire des obstacles au changement. Pour illustrer ceci, considérons successivement, avant de clore notre présentation, deux obstacles institutionnels au changement: l'institution du quasi-marché et l'institution de l'évaluation traditionnelle, certificative.

\section{Le quasi-marché comme mode institutionnalisé de régulation}

La liberté d'enseignement est un principe organisateur majeur du système scolaire en Communauté française, notamment la liberté accordée aux parents pour choisir l'école de leurs enfants. Cette liberté de choix, instituée au départ pour organiser une transaction entre milieux sociaux catholiques et laïcs, et coulée dans plusieurs textes clés du système juridico-politique (Constitution, Pacte scolaire), tend depuis une trentaine d'années à changer de signification, sous l'influence notamment de la forte sécularisation du pays. Elle devient de plus en plus une liberté «marchande», permettant aux parents de choisir l'école selon des critères renvoyant prioritairement à la «qualité d'enseignement» ou à des opportunités personnelles. Le système scolaire belge se rapproche ainsi d'un «quasi-marché» (Vandenberghe, 1998), comme en Angleterre ou en Nouvelle-Zélande, sans pour autant que ce soit le résultat d'une politique volontariste et consciente.

Les logiques d'action des établissements découlent partiellement de la position qu'ils occupent au sein du quasi-marché, dont les chefs d'établissement doivent nécessairement tenir compte. Il s'agit d'un principe de réalité. Un chef d'établissement informé des résultats de recherche sur l'hétérogénéité des classes et convaincu du caractère positif de celle-ci hésitera sans doute avant de composer des classes plus hétérogènes, s'il risque de s'aliéner une partie des enseignants et de perdre des élèves dont les parents n'apprécieraient pas la nouvelle politique de constitution des classes. Le 
chef d'établissement, c'est son lot quotidien, est soumis à de multiples pressions parfois opposées et entre lesquelles il lui revient d'arbitrer.

À ce propos, force est de constater l'absence d'entrepreneurs officiels de changement institutionnel au niveau de l'organisation scolaire. En fait, les chefs d'établissement, désormais encouragés à devenir des «managers» ou des «pilotes» du changement (Tilman et Ouali, 2001), sont progressivement appelés à remplir eux-mêmes ce rôle d'entrepreneur de changement dans leur établissement. L'entreprise de changement institutionnel au niveau de l'établissement relève donc de la contingence. Elle dépend de la présence ou non d'un chef d'établissement mobilisé sur le plan pédagogique ou qui a intériorisé ce nouveau rôle managérial.

Or, compte tenu du contexte institutionnel du système scolaire belge, le loyalisme à l'égard de la régulation politique requiert des conditions de réalisation spécifiques et assez sélectives qui — nous en faisons l'hypothèse — ne se rencontrent probablement pas fréquemment. En effet, dans un contexte de concurrence entre établissements, tout incite le dirigeant local (se comportant en «bon manager») à privilégier les intérêts de son établissement et de ses collectifs, parfois au détriment de la lettre ou de l'esprit de la loi.

En conséquence, le processus de réception et de mise en œuvre de la réforme dans les établissements se caractérise par une diversification, voire un éclatement dans les modes de réception de la politique d'éducation qui, in fine, produit un écart entre les intentions politiques et leur mise en pratique. C'est à l'échelle de l'établissement scolaire que s'effectue actuellement la mise en cohérence des politiques, des contraintes et des ressources environnementales locales, par un travail d'appropriation, de détournement de sens, voire de mobilisation des ressources et des principes de la réforme dans une perspective stratégique.

\section{L'évaluation traditionnelle certificative ou l'institution de la note}

Le fait que certains élèves semblent nettement réticents à s'investir dans l'apprentissage dès lors que le passage de classe devient automatique révèle, on l'a vu, un rapport instrumental à la scolarité. Ce qui motive les élèves à travailler est le système de notation, lié aux modes traditionnels d'évaluation scolaire.

La réforme propose de substituer à la notation, cet équivalent scolaire de la carotte et du bâton, d'autres modes de motivation des élèves. Toutefois, lorsqu'ils ont été soumis à un tel régime pendant plusieurs années, il s'avère difficile d'intéresser les élèves au savoir pour le sens qu'il donne à la réalité, pour l'enrichissement personnel qu'il apporte, pour la satisfaction de l'esprit qu'il favorise (Perrenoud, 1998). Au fond, l'élève autonome n'apparaît pas par génération spontanée, il est tributaire des institutions (Lahire, 2005). Jusqu'à présent, étant donné ce système institutionnalisé de la note, l'élève autonome demeure largement virtuel.

Dans la mesure où l'obéissance et la mobilisation des élèves au travail passaient jusqu'ici largement par le droit accordé à l'enseignant d'autoriser ou d'interdire la progression des élèves d'une année à l'autre, il n'est manifestement pas simple pour lui de 
renoncer au redoublement du jour au lendemain. Les conditions de travail en classe et l'institutionnalisation des rapports entre enseignants et élèves tendent donc à délégitimer la réforme.

\section{CONCLUSIONS: LES CONDITIONS DE PLAUSiBILITÉ AU CONSENTEMENT}

Nous avons proposé dans cet article de lire la politique de lutte contre le redoublement menée ces quinze dernières années en Communauté française de Belgique comme participant d'un processus de changement institutionnel.

Ce dernier, approché sous un angle cognitif, a été envisagé comme un processus de construction d'une légitimité des dispositifs promus par la loi, processus ouvert et incertain qui se joue de façon conflictuelle et non linéaire sur plusieurs scènes sociales. Favorisés par un contexte politique, social et économique et par d'autres événements déclencheurs, des entrepreneurs de changement institutionnel cherchent intentionnellement ou non à établir la légitimité cognitive, morale et pragmatique de la réforme. Ce faisant, ils rencontrent une normativité établie, des obstacles matériels ou cognitifs, des actions organisées qui tendent à fonctionner comme des entreprises de stabilité institutionnelle, remettant en cause l'une ou l'autre forme de légitimité de la réforme.

Au total, si l'on accepte que l'école constitue une macro-institution, composée de nombreuses règles et pratiques qui sont elles-mêmes des institutions, on peut considérer que la politique de lutte contre le redoublement en Belgique francophone illustre les limites et la complexité de toute entreprise politique de transformation institutionnelle de l'école.

À rebours de l'idée naïve qui lie nécessité (et donc possibilité) de changement institutionnel à une crise d'efficacité des systèmes, l'enquête montre que le changement ne résulte pas mécaniquement de la perception des dysfonctionnements, et qu’il se joue de façon décisive dans les établissements scolaires ou dans les classes à l'occasion de la mise en œuvre de la politique. Le caractère essentiel des espaces locaux et de la légitimité pragmatique pour obtenir le consentement des acteurs de terrain apparaît sous-estimé, tant sur le plan théorique par l'analyse cognitive des politiques publiques et par les néo-institutionnalistes que sur le plan politique par les responsables de l'action publique en matière d'enseignement. En ce sens, une des implications de notre analyse sur laquelle nous souhaitons insister pour conclure est la nécessité de penser les conditions de plausibilité des réformes.

Sous quelles conditions une tentative politique de changement institutionnel conduit-elle à une institutionnalisation effective? La théorie institutionnelle ne s'est guère intéressée à ce genre de questions. Le plus souvent, les auteurs s'interrogent seulement sur l'amont, sur les conditions qui rendent possible l'introduction d'un changement institutionnel et non sur sa réception et sa mise en œuvre, ses conditions de recevabilité et de praticabilité. La raison en est souvent que les modèles ne s'intéressent pas aux changements institutionnels initiés par d'autres acteurs que les participants à l'institution en question, comme c'est précisément le cas lorsque le changement est initié par une réforme politique, qui procède selon une méthode descendante de 
type top-down. Qu'arrive-t-il lorsque le mouvement de réforme institutionnelle impulsé par l'État, se faisant entrepreneur institutionnel, n'est pas souhaité par la majorité des intéressés? Afin de le savoir, nous avons pris en considération le processus parallèle de désinstitutionnalisation des formes et des pratiques institutionnalisées. Les interactions, tensions et conflits entre processus et acteurs de changement et de nonchangement ont été placés au cour de l'analyse. Prendre en compte les dynamiques contradictoires suppose de lier chaque concept à son envers et de les envisager dans une logique d'équivalence au plan analytique. Ainsi, aux stratégies de légitimation correspondent les stratégies de dénigrement et de déstabilisation ou de délégitimation; aux entrepreneurs de changement institutionnel répondent des entrepreneurs de nonchangement, etc. Nous avons de la sorte rendu les propositions des néo-institutionnalistes plus dynamiques et plus interactionnistes qu'elles ne le sont habituellement dans la théorie.

Les obstacles institutionnels à la réforme et au changement pédagogique demeurent aussi largement des impensés politiques. La question du consentement des acteurs de terrain est trop souvent réduite à un simple problème de représentations sociales de la part des enseignants, représentations plus ou moins justes ou erronées et plus ou moins en phase ou en décalage par rapport à la philosophie qui sous-tend la réforme. La légitimation cognitive et normative est certes nécessaire mais nullement suffisante. Encore faut-il construire la légitimité pragmatique, c'est-à-dire mettre en place les conditions institutionnelles qui rendent recevables et praticables le changement et les nouvelles idées qui l'accompagnent. Cette analyse suggère que le cognitivo-normatif et le pragmatique sont intimement connectés, les idées abstraites doivent trouver un écho dans l'expérience concrète et quotidienne des acteurs. Autrement dit, il s'agit d'envisager le cognitif de manière plus large, pour y inclure aussi les dispositifs cognitifs socialement partagés que forment les institutions. Construire la légitimité pragmatique commande de changer les institutions et non seulement les individus (Douglas, 1999). À cet égard, ce qui semble avoir empêché un réel changement institutionnel, c'est notamment une prise de conscience de la part des responsables politiques que «tout se tient» dans le système éducatif (Hutmacher, 1993) et que le redoublement est encastré dans un réseau de règles institutionnelles.

Cet oubli de la dimension institutionnelle est typique, selon De Munck, de la société moderne qui a tendance à percevoir ses apprentissages sociaux comme des apprentissages individuels: «Dans cette perspective "atomiste" sur le social, le savoir est localisé dans la tête des individus, et les institutions dépendent de leurs intentions. Le problème de cette petite "philosophie de l'esprit" à usage politique est qu'elle rend systématiquement invisible l'opération effective des dispositifs cognitifs collectifs, et leur contribution non seulement aux échecs, mais aussi aux succès des apprentissages individuels. Lorsque les normes sont réduites à des contrats inter-individuels, et les pouvoirs à des capacités personnelles, il devient impossible d'analyser et d'évaluer les contextes collectifs, a fortiori de les transformer. La direction des apprentissages collectifs 
est alors laissée aux mains de forces immaîtrisées, et ne peut plus faire l'objet d'une réappropriation publique et explicite» (De Munck, 1999: 196).

Sans doute ne faut-il pas surestimer la rationalité de la politique publique, qui n'a pas exclusivement pour vocation de résoudre des problèmes. Cette question de la rationalité de l'action publique en éducation mériterait en tout cas d'être approfondie. Des enquêtes diachroniques sur une plus longue période seraient nécessaires, notamment afin d'étudier précisément comment les processus d'apprentissage politique influencent la manière de concevoir et de légitimer l'action publique. La construction de la légitimité pragmatique implique certainement aussi la prise en compte des effets des politiques antérieures.

\title{
RÉSUMÉ
}

Cet article invite à lire les politiques scolaires comme des entreprises de changement institutionnel. L'approche cognitive adoptée conduit à placer la légitimation au cœur du processus de changement institutionnel. L'analyse est menée à partir de l'étude d'une politique de lutte contre le redoublement en Belgique francophone. Ce cas, qui s'est soldé par un échec relatif, illustre les limites et la complexité de toute entreprise politique de transformation institutionnelle de l'école. Alors que la réforme s'appuie sur l'expertise pédagogique et économique pour légitimer la suppression du redoublement, l'enquête montre que le changement institutionnel ne résulte pas mécaniquement de la perception de dysfonctionnements, mais nécessite le consentement des acteurs scolaires locaux, dans la mesure où le changement se joue de façon décisive dans les établissements scolaires et dans les classes. À cet égard, l'enquête souligne l'importance, tant théorique que pratique, de l'encastrement institutionnel du redoublement, encastrement qui mine la légitimité pragmatique de la réforme sur le terrain.

\begin{abstract}
This article invites its readers to view education policies as enterprises for institutional change. The cognitive approach adopted leads us to place legitimating at the core of the institutional change process. The analysis is based on the study of the combat against grade repetition in French-speaking Belgium. This issue, which ended in relative failure, illustrates the limits and the complexity of any political enterprise for institutional transformation in education. While the reform is founded on pedagogical and economic expertise to legitimate the abandon of grade repetition, the fieldwork reveals that institutional change does not automatically follow the perception of dysfunctions but requires the approval of local educational players as the actual change takes place in school and in class. For this reason the study highlights the importance, both theoretical and practical, of the institutional framework of grade repetition, a framework that undermines the pragmatic legitimacy of reform in the field.
\end{abstract}

\section{RESUMEN}

Este artículo invita a leer las políticas escolares como proyectos de cambio institucional. El enfoque cognoscitivo adoptado conduce a colocar la legitimación en el centro del proceso de cambio institucional. El análisis se lleva a cabo a partir del estudio de una política de lucha contra la repetición de clases en la Bélgica francófona. Este caso, que resultó un fracaso relativo, 
ilustra los límites y la complejidad de toda empresa política de transformación institucional de la escuela. Mientras que la reforma se basa en el dictamen pedagógico y económico para legitimar la supresión de la repetición de clases, la investigación pone de manifiesto que el cambio institucional no resulta mecánicamente de la percepción de las disfunciones, sino que requiere el consentimiento de los protagonistas escolares locales, en la medida en que el cambio se juega de manera decisiva en los establecimientos escolares y en las clases. A este respecto, la investigación destaca la importancia, tanto teórica como práctica, del ajuste institucional de la repetición de clases, ajuste que mina la legitimidad pragmática de la reforma en la práctica.

\section{BIBLIOGRAPHIE}

Archer, M. (2004). «Entre la structure et l'action, le temps», Revue du MAUSS, n 24, p. 329-350.

Boulad-Ayoub, J. (2003). «Légitimité, légalité et vie politique», in J. Boulad-Ayoub et L. Bonneville, Souverainetés en crise, Québec, L'Harmattan/Presses de l'Université Laval, p. 71-80.

Boussaguet, L., S. Jacquot et P. Ravinet (2004). Dictionnaire des politiques publiques, Paris, Presses de Science Po.

Buisson-Fenet, H. (2007). «L'éducation scolaire au prisme de la science politique: vers une sociologie politique comparée de l'action publique éducative», Revue internationale de politique comparée, vol. 14, $\mathrm{n}^{\circ} 3$.

Cattonar, B. (2005). L'identité professionnelle des enseignants du secondaire: approche biographique et contextuelle, Thèse de doctorat, Université catholique de Louvain.

CrahaY, M. (1992). Échec des élèves, échec de l'école? La Communauté française de Belgique en échec scolaire, Bruxelles, Rapport au Conseil de l'Éducation et de la Formation.

De Munck, J. (1999). L'institution sociale de l'esprit: nouvelles approches de la raison, Paris, PUF.

Derouet, J.-L. (2000). «Une science de l'administration scolaire est-elle possible?», Revue Française de Pédagogie, $\mathrm{n}^{\circ} 130$, p. 5-14.

DiMaggio, P. (1988). «Interest and Agency in Institutional Theory», in L. ZucKer (éd.), Institutional Patterns and Organizations, Cambridge, Ballinger, p. 3-22.

Douglas, M. (1999). Comment pensent les institutions, Paris, La Découverte.

Draelants, H. (2008). «Les fonctions latentes du redoublement», Éducation et Sociétés, n 21, p. 163-180

Draelants, H. (2007). «Les savoirs pédagogiques comme source de légitimation pour l'action publique en éducation ", Cahiers de recherche en éducation et formation, $\mathrm{n}^{\circ}$ 59, p. 3-33.

Draelants, H. (2006). Politiques d'éducation et changement institutionnel: le cas de la réforme du premier degré de l'enseignement secondaire en Belgique francophone, Thèse de doctorat, Université catholique de Louvain.

Dubet, F. (2002). Le déclin de l'institution, Paris, Seuil.

Dupriez, V. et J. CoRnet (2005). La rénovation de l'école primaire: comprendre les enjeux du changement pédagogique, Bruxelles, De Boeck Université.

Ferréol, G. (dir.) (1995). Dictionnaire de sociologie, Paris, Armand Colin.

Frenay, M. et C. Maroy (éd.) (2004). L'école, 6 ans après le décret «missions», Presses universitaires de Louvain, Louvain-la-Neuve.

Greenwood, R., R. Suddaby et C.R. Hinings (2002). «Theorizing Change: The Role of Professional Associations in the Transformation of Institutionalized Fields", Academy of Management Journal, vol. $45, \mathrm{n}^{\circ} 1, \mathrm{p} .58-80$.

Hall, P.A. et R.C.R. TAYlor (1997). «La science politique et les trois néo-institutionnalismes», Revue française de science politique, vol. 47, $\mathrm{n}^{\circ} 3-4$, p. 469-496.

Hassenteufel, P. et A. Smith (2002). «Essouflement ou second souffle? L'analyse des politiques publiques “à la française”", Revue française de science politique, vol. 52, nº 1, p. 53-73. 
Hutmacher, W. (1993). «Quand la réalité résiste à la lutte contre l'échec scolaire: analyse du redoublement dans l'enseignement primaire genevois", Cahier du Service de la Recherche Sociologique, nº 33, p. 3-165.

JAVEAU, C. (2001). «Le savant et le politique revisités: les rapports d'aujourd'hui entre les champs politique et scientifique», Revue de l'Institut de Sociologie, n 1-4, p. 167-176.

Kingdon, J.W. (1984). Agendas, Alternatives and Public Policies, Boston, Little, Brown and Co.

LAHIRE, B. (2005). L'esprit sociologique, Paris, La Découverte.

Le BART, C. (1998). Le discours politique, Paris, PUF, coll. «Que Sais-je?».

Mangez, E. (2002). «Régulation de l'action éducative dans les années quatre-vingt-dix», Éducations et Sociétés, no 8 , p. 81-96.

Maroy, C. (dir.) (2006). École, régulation et marché, Paris, PUF.

Maroy, C. et V. Dupriez (2000). «La régulation dans les systèmes scolaires: proposition théorique et analyse du cadre structurel en Belgique francophone», Revue Française de Pédagogie, nº 130, p. 73-87.

Muller, P. et Y. Surel (1998). L'analyse des politiques publiques, Paris, Montchrestien.

Perrenoud, P. (1998). L'évaluation des élèves: de la fabrication de l'excellence à la régulation des apprentissages. Entre deux logiques, Bruxelles, De Boeck Université.

Powell, W.W. et P.J. DiMaggio (éd.) (1991). The New Institutionalism in Organizational Analysis, Chicago, University of Chicago Press.

RADAELLI, C.M. (2000). «Logiques de pouvoirs et récits dans les politiques publiques de l'Unioneuropéenne», Revue française de science politique, vol. 50, $\mathrm{n}^{\circ} 2$, p. 255-275.

Radioscopie de l'enseignement en Communauté française de Belgique (1992). Les dépenses de personnel dans l'enseignement fondamental et secondaire de la Communauté française, Contribution de l'IRES, Rapport final, UCL.

Rémy, J., L. Voyé et E. Servais (1978). Produire ou reproduire? Tome 1: conflits et transaction sociale, Bruxelles, Vie Ouvrière.

Scotт, W.R. (1995). Institutions and Organizations, Thousand Oaks, Sage.

SEO, M.-G. et W.E.D. Creed (2002). «Institutional Contradictions, Praxis, and Institutional Change: A Dialectical Perspective», Academy of Management Review, vol. 27, n 2, p. 222-247.

Suchman, M.C. (1995). «Managing Legitimacy: Strategic and Institutional Approaches», Academy of Management Review, vol. 20, $\mathrm{n}^{\circ}$ 3, p. 571-610.

Théret, B. (2000). «Nouvelle économie institutionnelle, économie des conventions et théorie de la régulation: vers une synthèse institutionnaliste?», La lettre de la régulation, $\mathrm{n}^{\circ} 35$.

Tilman, F. et N. Ouali (2001). Piloter un établissement scolaire: lectures et stratégies de la conduite du changement à l'école, Bruxelles, De Boeck Université.

van Campenhoudt, L. et A. Franssen (2004). La consultation des enseignants du secondaire, Rapport élaboré pour la Commission de Pilotage, Ministère de la Communauté française.

VANDEnberghe, V. (1998). «L'enseignement en Communauté française de Belgique: un quasi-marché», Reflets et perspectives de la vie économique, vol. $37, \mathrm{n}^{\circ} 1, \mathrm{p} .65-75$.

van HaEcht, A. (2004). «Histoire et critique en sociologie de l'éducation: le cas de la Communauté française de Belgique», Éducation et Sociétés, nº 13.

VAN HAECHT, A. (1998). «Les politiques éducatives, figure exemplaire des politiques publiques?», Éducation et Sociétés, $\mathrm{n}^{\circ} 1$, p. 21-46.

van Zanten, A. (2004). Les politiques d'éducation, Paris, PUF, coll. «Que sais-je?». 